\title{
Combining Forecast of Electricity Price with Adaptive Weights Selected by SOM Clustering
}

\author{
Yi Feng \\ State Grid co. Itd, No 86, West Changan Street, 100031, \\ Beijing, China \\ Yifeng1358@126.com
}

\begin{abstract}
This paper proposes an efficient method of combining modelling to forecast day-ahead electricity price. The basic idea of this method is to develop a robust technique to select the proper combining weights of individual models according to their historical performance on similar circumstances. Firstly individuals were trained and validated separately, and then self-organising map neural network (SOM) was applied to cluster the circumstance into multiple categories. For day-ahead price forecasting, the circumstances of the coming day were compared with those of past days and then clustered into the same category by SOM. The combining weights of individual models for next-day price forecasting were determined by their performances in same category in the past. The final forecast was a weighted sum of the individual predictions with the time-varying weights obtained above. An experimental study indicated that the proposed method outperforms other price-forecasting techniques with high efficiency more robustness.

Keywords: electricity price forecasting; Combining forecast; SOM; Similar days clustering
\end{abstract}

\section{Introduction}

Recent years various techniques have been developed for model price analysis and forecasting in electricity market. Among these techniques, stochastic time series modelling and artificial intelligence (AI) modelling are most commonly used. Time-series modelling exploits information contained in historical electricity prices to mine the linear or nonlinear map relations between the future price and 
historical prices[1]. However, this technique pays less attention to external influences. AI modelling is a powerful method for inferring hidden mapping in data[2], but its performance relies on the tuneable parameters or inputs. On the other hand, efforts have been made to combine the forecasts by the two methods[3].

Researchers have made progress on different aspects of the technique and can make full use of the forecasting ability of diversification models[4, 5]. These techniques include liner combining, such as simple average (SA) and nonlinear combining[6]. Among these techniques, SA with equal weights is the simplest, and most of time it outperforms individual models, even other sophisticated combining methods. It is suggested that SA is preferred for combining[7, 8,9].

The combining weights in the modelling of traditional combining techniques are usually obtained according to the overall historical performance of the individuals. Nevertheless the performance of the individual model fluctuates under different circumstances, sometime one model outperforms others, and sometime it performs worse. Thus, the generalization abilities of the models cannot be represented by fixed weights usually.

The combining with varying weights according to the historical performances under similar circumstance can be expected better than that with the fixed weights. The problem is how to find out the historical similar circumstances among numerous data for a target hour. Self-organizing map neural network (SOM) is a classic clustering artificial neural network (ANN) that has been widely applied for pattern recognition [10]. In[11], SOM was applied to cluster the input data set into several subsets in which a group models were used to fit and forecast separately. Inspired by [11] we use SOM to classify the historical input data set into multiple patterns, and then analyse the forecasting abilities of the individual models (time-series modelling and ANN modelling) under these patterns. When we forecast the future price with combining technique, we choose the weights of individuals by their previous performance within the same pattern. The remainder of the paper is organized as follows. In Section 2, we describe the basic ideas of SOM and their application to combined forecasts and the proposed combining modelling process; Original data for experiments and results of individual forecasts are analyzed in Section 3; the performance of the proposed method is compared with the individual models as well as SA in Section 4; Finally, a 
discussion of the results and the conclusions drawn from the study are presented in Section 5.

\section{APPROACH OF COMBINING FORECASTS WITH SOM}

SOM is a classic unsupervised algorithms used to classify patterns automatically. The network interconnected processing units competes to learn the input vectors by changing their weights to be closer in value to the inputs. The principles of SOM will not be discussed in detail here because they have been described in other reports[10].

Suppose that there are $\mathrm{m}$ individual forecasts by model set; we want to combine them to obtain a more reliable prediction. We use external information, such as historical prices and historical loads, to cluster the circumstances. The weights of individual models to be combined are determined according to their historical performances under similar circumstances. The hourly price varies from hour to hour, and therefore, 24 models were built to forecast the hourly prices in this study. We will take one hour as an example to discuss the modelling approach below. The relative error and mean absolute percentage error are used to evaluate the performances of the model.

There are eight steps in the combining modelling procedure:

Step 1. Initiation. Selecting the individual candidate models.

Step 2. Classification. Classifying the data into the training, validation and test sets.

Step 3. Training. Training the candidate models with the training data set.

Step 4. Validation and Testing. Input the validation and test sets into the trained models to obtain the validation error $\mathrm{e}_{i j k}$ and forecasting price $\mathrm{P}_{i j k}$.

Step 5. Clustering. Clustering the data into different categories of similar days with SOM.

Step 6. Selecting the combining weights. For any hour of the next day in which the price is to be forecasted, we search for a similar circumstance among the historical data and then calculate MAPE of the similar days to obtain the combining weights $w_{k}$, which are calculated by the harmonic mean (which is typically used to calculate the average rate) of MAPE $k$, as described by (1). 


$$
w_{k}=\frac{\left|1 / \operatorname{MAPE}_{k}\right|}{\sum_{k=1}^{m}\left|1 / \operatorname{MAPE}_{k}\right|}
$$

where MAPEk is the MAPE by the $k^{\text {th }}$ model of similar days determined, $k=1,2$, m.

For any hourly price of the next day, if there is no identical circumstance category, equal weights will be adopted for the combining forecasting.

Step $7 . \quad$ Combining forecasts. The forecasts from different candidates are combined to get the final forecast yij for the ith hour of the jth day with the weights determined in Step 6, as (2) shows.

$$
y_{i j}=w_{k} \times P_{i j k}
$$

\section{ORIGINAL DATA FOR THE EXPERIMENT}

In this paper, peak price in August of the PJM day-ahead electricity market in 2010 were used in the experiments. The data from the previous two months served as training and validation sets, respectively.

In this section, the individual modelling part of the modelling process. Firstly, the data for the experiment are analyzed and divided into training, validation and test sets as discussed in Section 3; and then the candidate models are trained and validated. Ultimately, the validation errors, predicted prices and test errors are obtained.

As discussed previously, time-series modelling and AI modelling exploit information that influences price differently. Two classic AI modelling techniques, Support Vector Machine (SVM), generalized regression neural network (GRNN), and an efficient time-series model Generalized AutoRegressive Conditional Heteroskedasticity (GARCH) are selected as individual candidates. Additionally, a model from a direct-way, day-ahead model (DA) is selected as a candidate to reduce the entire correlation among the individual models.

DA modelling, which is a direct genre of modelling, is adopted to alleviate the correlation of the candidate individuals. In this model, the price of the target hour on the previous day is taken as the forecast price with consideration of weekly seasonality. 
Table 1 presents the parameters of the individual modes. With the parameters selected in Table I, we build the individual models with the training set and then obtain the validation error, The MAPE of validation by the above four models are $8.54 \%, 11.60 \%, 12.65 \%$ and $13.55 \%$ respectively, showing that SVM has the best performance.

Table 1 Parameters of the candidate models

\begin{tabular}{l|l|l}
\hline Model & Parameter & Value \\
\hline SVM & C, nu, e, g & $200,0.2,0.00002,0.004$ \\
\hline GRNN & r & 0.05 \\
\hline GARCH & r, m, p, q & $2,1,1,1$ \\
\hline DA & - & - \\
\hline
\end{tabular}

\section{COMPARISON BETWEEN combing model AND THE INDIVIDUAL MODELS}

In this section, a combining model with SOM clustering algorithm is developed and compared with the individual models. The combining weights of the individual models are calculated according to their performance under different circumstances classified by the SOM.

The input of the SOM is the same as that of the SVM and GRNN, and the firstand second-layer dimensions of the SOM are set to 4. Using the combining weights and individual forecasts, we obtain the combining forecast. The SVM model and SOM combining model provide better results than the other models. When MAPE is low, the performance of SOM and SVM is similar; as MAPE rises, SOM shows high accuracy, but when MAPEs by most individual model are not so good.

A further study of comparison between the SOM combining and SA is fulfilled. Six other models (three other SVMs and three other GRNNs with different parameters) are appended into the pool of the above four models to augment the candidate pool for combining forecasting. For the ten individuals, there are 1024 combinations (the 10th power of two). For each combination the SA and SOM are used to calculate the combining forecast. A gap of the MAPE by the SA and SOM combining method is calculated to evaluate the advantage of SOM against SA. The performance comparison between the SA and SOM on the 1024 combinations is presented in Figure 1. 
It can be seen from Figure 1 that nearly all the gap of MAPE between the two methods are no less than zero although it fluctuates all the time, which means that the MAPEs by SA are bigger than SOM all the time. This confirms the guess that the SOM is more efficiency and robustness than SA. With the number of combinations increases (after 4), the gap between the two models decrease, which means that with more combining candidates, the MAPE of SOM has a trend of approaching the MAPE of SA.

In fact comparison between the two combining model on the first 11 combinations is meaningless, as the first one is the combination of null individual, and the following 10 are in the combination form that has only one individual model. Among the 1013 combinations (1024-11=1013), the proper number of combination is 4 for where MAPE are smaller than other number and the gap between SA and SOM are bigger.

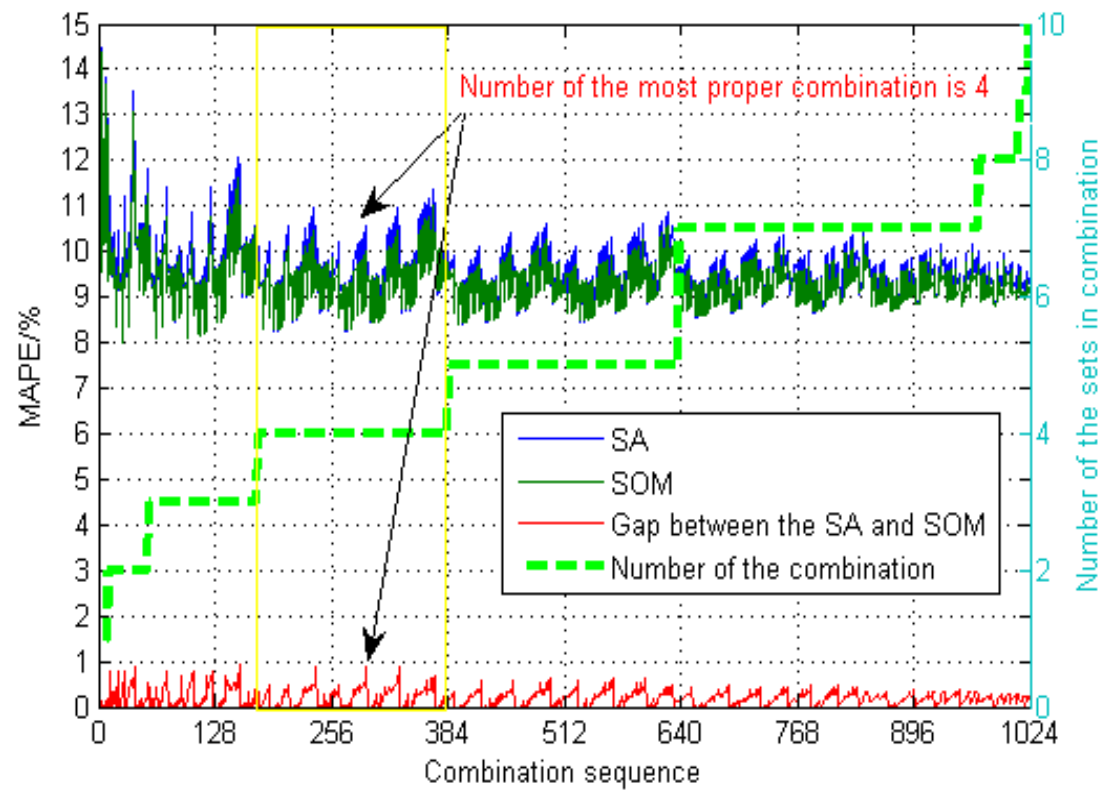

Figure 1. Performance of different combinations by the SA and SOM combining

\section{DISCUSSION AND CONCLUSIONS}

In this paper, we developed a combined method with SOM to forecast the day-ahead price of the PJM market. The purpose of this paper is to develop a novel 
combining model for day-ahead price forecasting, whose weights are time-varying and chosen by similar-day clustering techniques. A comparison with the classic combining method, SA shows the proposed method has the virtues of efficiency and robustness. Experiment shows that it outperforms its counterparts with more robust performance. The research contributes to find proper combining weights for a more robust general forecast; it also enriches the experiments of electricity price forecast where combining forecast is quite limited. In the process of determining the combining weights of the individual modes, only the MAPEs were exploited; other information, such as the forecast trend and error skewness, was not considered here. Further study will focus on how to improve the accuracy of the combining forecast while keeping its robustness.

\section{References}

[1] Z. Tan, J. Zhang, J. Wang and J. Xu, Day-ahead electricity price forecasting using wavelet transform combined with ARIMA and GARCH models, Applied Energy. 87, 11 (2010)3606-3610.

[2] G. C. S. Lui, W. K. Li, K. M. Y. Leung, J. H. W. Lee and A. W. Jayawardena, Modelling algal blooms using vector autoregressive model with exogenous variables and long memory filter, Ecological Modelling. 200(2007)130-138.

[3] G. R. Reeves and K. D. Lawrence, Combining forecasts given different types of objectives, European Journal Of Operational Research. 51, 1(1991) 65-72,.

[4] G. R. Reeves, K. D. Lawrence, S. M. Lawrence and J. B. Guerard Jr., Combining earnings forecasts using multiple objective linear programming, Computers and Operations Research. 15, 6(1988) 551-559.

[6] S. Lessmann, M. Sung, J. E. V. Johnson and T. Ma, A new methodology for generating and combining statistical forecasting models to enhance competitive event prediction, European Journal Of Operational Research. 218, 1(2012).

[7] R. L. Winkler, Combining forecasts: A philosophical basis and some current issues, International Journal of Forecasting. 5, 4(1989), 605-609.

[8] V. Genre, G. Kenny, A. Meyler and A. Timmermann, Combining expert forecasts: Can anything beat the simple average? International Journal of Forecasting . 29, 1(2013)108-121,201.

[9] R. T. Clemen, Combining forecasts: A review and annotated bibliography, International Journal of Forecasting . 5, 4(19689), 559-583 
[10] M. Cottrell, J. C. Fort and G. Pagès, Theoretical aspects of the SOM algorithm, Neurocomputing. 21, 1(1998) 119-138.

[11] D. Niu, D. Liu and D. D. Wu, A soft computing system for day-ahead electricity price forecasting, Applied Soft Computing, 10(2010) 868-875. 